\title{
Evaluation in Diagnosis and Management of Diabetic Neuropathy
}

\author{
Wendy Waldman Zadeh ${ }^{1,2^{*}}$, Pichit Thanupakorn ${ }^{3}$ \\ ${ }^{1}$ Broadlawns Medical Center, Des Moines, USA \\ ${ }^{2}$ University of Iowa, Iowa City, USA \\ ${ }^{3}$ University of Iowa Hospitals \& Clinics, Iowa City, USA \\ Email: ^wwaldman@Broadlawns.org, Pichit-thanupakorn@uiowa.edu
}

How to cite this paper: Zadeh, W.W. and Thanupakorn, P. (2021) Evaluation in Diagnosis and Management of Diabetic Neuropathy. Journal of Diabetes Mellitus, 11, 348-353.

https://doi.org/10.4236/jdm.2021.115027

Received: September 22, 2021

Accepted: November 15, 2021

Published: November 18, 2021

Copyright $\odot 2021$ by author(s) and Scientific Research Publishing Inc. This work is licensed under the Creative Commons Attribution International License (CC BY 4.0).

http://creativecommons.org/licenses/by/4.0/ (c) (i) Open Access

\begin{abstract}
The 100-year anniversary of insulin is explored by focusing on diabetic neuropathy. Neuropathy is so common to diabetes, it is well described even in the earliest accounts of diabetes. This article reviews the most common neuropathy syndromes, and the consensus of effective treatment for neuropathic pain. Pharmacological advances in neuropathy are still largely focused on pain control, not neuropathy intervention. The article reviews the established and lesser tested therapies used for pain control. It also reviews the pathophysiology of the disease state, including the many factors and steps that culminate to produce neuropathy and its different iterations. In the future, new ways to treat diabetic neuropathy may be geared toward treating specific pathophysiological step-points on the way to nerve damage. In the future, prevention and a deeper look at the impact of socioeconomic status as a predictor of diabetes will hopefully encompass a bigger part of pre-diabetic care.
\end{abstract}

\section{Keywords}

Diabetic Neuropathy, Glycemic Control, Glycation End Products,

Neuropathy Pain Medications, Preventive Medicine, Socioeconomic Status

As we reflect on 100 years of insulin, diabetes and diabetic neuropathy, history describes the impact of the disease as early as writing is available. Ancient papyrus texts as far back as $1550 \mathrm{BC}$ record symptoms of diabetes. Regarding diabetic neuropathy, in the 5th century AD there is a Sanskrit recording of "burning in the palms and soles," with other descriptors of profound thirst and "sweet urine" that paints a picture of what certainly must have been diabetes and accompanying neuropathic symptoms [1]. Autonomic dysfunction manifesting as impo- 
tence associated with extreme thirst among other symptoms was described in a Japanese man Michinaga around 1000 AD. In this case, procreation concerns were as important as any other inconvenience since his family lineage maintained the Imperial House [2]. Yet a clear link between diabetes as a neuropathic syndrome was not well documented until the 1800s [3] [4]. Currently, the general rule is that peripheral diabetic neuropathy will affect $50 \%$ of patients with diabetes. Not to understate its annoyance, it is considered a major cause of disability among diabetics and is associated with a poor quality of life [5].

Once insulin was discovered, a more systematized approach was taken to tie diabetes to its pathological offshoots, hence the well-known entity of diabetic neuropathy. By 1960, all assortments of diabetic neuropathy were outlined and clearly characterized. The most common neurological complication is a pure sensory syndrome, considered a small fiber neuropathy, which manifests most frequently as the feet feeling cool and painful, especially at night. Sometimes this occurs with detectable loss of pain (pin prick) and temperature fibers, and other times this is seldom found. The findings affect longer nerves first, which is why the symptoms frequently start in the feet. Non-myelinated nerve fibers are the first to suffer injury and therefore pain and temperature is first impacted as well as the exam finding of lost reflexes. Autonomic nerves are also susceptible to diabetic neuropathy. Symptoms here have included paroxysmal diarrhea, fecal incontinence, erection or libido loss and impotence, bladder paralysis, loss of sweating, postural hypotension, postural tachycardia, pupil changes and gastric retention. Separate from neuropathy, a diabetic amyotrophy is also well described. This manifests as an asymmetrical weakness of an arm or a leg. This is purely a motor loss syndrome. Mixed sensory and motor diabetic syndromes are, too, reported, although these are largely experienced as a sensory syndrome, even while motor endplates show some effect [6]. In typical cases of polyneuropathy, EMG/NC studies can help determine the presence and severity of a large fiber polyneuropathy. However, this information is not likely to alter patient management [7].

Even while intensive blood glucose control is the cornerstone of treatment, what has partly confused the treatment of diabetic neuropathy over the years has been the oft reported disconnect between glycemic control and neuropathic manifestations [8]. Not only is a connection to glycemic control and neuropathy inconsistently forecasted, neuropathic manifestations and progression are not reliably predictable, although consistently poor control of diabetes will almost assuredly hasten the worsening of neuropathy. Ultimately, data review suggests that enhanced glucose control prevents the development of neuropathy in type 1 diabetes. In type 2 diabetes, enhanced glucose control reduces the rate of neuropathy and reduces nerve conduction and vibration threshold irregularities, although the correlation is often considered modest. This lack of correlation may reflect the differing pathophysiology between the two diseases, which in turn point to different pathophysiological components within, even while the metabolic syndrome culminates similarly [9]. To complicate matters, there have been reports of acute painful "insulin neuritis" caused by rapidly achieved glycemic 
control [10]. What's more, frequent shifts in blood sugar, inside normal levels and outside it, may pose its own independent risk for neuropathy separate from more consistent glucose levels, even if these consistent levels run high [11]. Thus, it can be said that a direct cause and effect relationship between glucose control and neuropathy is lacking, particularly in type 2 diabetes. What we do know is that excess and varied glucose levels in the bloodstream manifests pathologically in multifactorial ways. Glycation end products begin a cascade of injury. When examined under the microscope, epineural and endoneural microangiopathy with endothelial cell hyperplasia and basement membrane thickening is frequently found. These changes along with arteriovenous shunting and hemorrhagic changes likely cause reduced blood flow to the nerves [12].

As the cascade continues, hyperglycemia-induced oxidative stress and resultant mitochondrial free radicals are found. These free radicals cycle back into the system to cause further damage. Hyperglycemia and lactate have also been described as directly triggering neuronal apoptosis. Malfunctioning glial cells and neuroinflammation are independent pathophysiological variables for neuronal cell damage. With blood flow reduction, there is alteration of affected redox status and an imbalance of calcium. All of the above factors have been implicated in nerve damage. Not only do nerve cells die more easily in a hyperglycemia setting, but repair pathways are also defective [13]. In experimental models, reduced neurotrophic factors are found as are insulin-like growth factors. As advances in pathophysiological understanding and biotechnology allow scientists a more precise and multi-leveled view and approach to diabetic neuropathy, those of us who spend most of our time in the clinic will attest to a sketchy correlation between nerve pain and finger-stick glucose logs. The degree of glycemic flux may be more important than overall blood sugar control [14].

Even while we understand better the complex networks involved in neuropathology, we as yet do not know how to intervene and halt these specifically damaging pathways. To date, medications that treat diabetic neuropathy are geared only toward symptomatic relief. For pain, level A data (as recommended by the American Academy of Neurology) exists for the symptomatic use of pregabalin alone. Level B data supports the use of duloxetine, gabapentin, venlafaxine and amitriptyline. Anticonvulsants, opioids, NMDA receptor antagonists, capsaicin, and antidepressants have been utilized, although benefits are varied with respect to pain control [15]. Other therapies may be tailored for the particular expression of the neuropathy. For example, metoclopramide is used for gastroparesis and phosphodiesterase type 5 inhibitors are used to treat erectile dysfunction [16]. Non-pharmacological treatments such as alpha-lipoic acid, acupuncture, reflexology, and Thai massage have been explored via a search database with some evidence of efficacy [17]. Additionally, there is some data to show that spinal cord stimulation and repetitive transcranial magnetic stimulation may have some utility as an adjuvant to conventional medical treatment. This will require further research [18]. No current therapies are proven to assist in preserving or healing nerve function. 
The question to ask as we now turn our heads toward the future is, what's in store for the next 100 years? Will therapies be available to improve or repair neuropathy specifically? Or is the answer to curing diabetic neuropathy found only in curing diabetes itself? The hope is that new neuropathy treatments will more precisely target what is being uncovered by way of neuropathophysiology. Since we now know there are differing and numerous mechanisms of actions at play, this would suggest that a multi-modality approach to pain control is needed. Still, it is likely that the central nervous system's important final pathways of pain processing will continue to be a mainstay approach to pain relief. Recent advances in the fields of neuroimaging and bioinformatics hold some promise to help target pain more precisely and thereby reduce therapeutic systemic side effects [13]. Nevertheless, medical treatments and technologies that tighten insulin control and thereby prevent glycosylated hemoglobin from damaging nerves will always be preferred to treatments that focus on pathology that is seemingly locked in place.

Herein lies the important and hopeful horizon of preventive medicine. Medicine is now more fully embracing ways to incorporate prevention into clinical care because data suggests that this indeed matters. An estimated $37 \%$ of prediabetic individuals may develop diabetes within four years, however, with interventions such as diet, exercise and BMI control, that percentage could decrease to $20 \%$ [19]. Where there is no diabetes, there is no diabetic neuropathy. Aggressive health prevention promises to bring with it a parade of neurological and general salubrity. Alzheimer's disease, many cancers, hypertension and high cholesterol (to name a few) are known diseased states whose numbers stand to significantly diminish if aggressive preventive measures are in place. With an eye toward the future, and with lessons learned from the past, prevention of disease must find its way into the medical field.

And finally, it would be remiss to not take notice of how socioeconomic status within North America plays a role in insulin resistance, diabetes, and resulting diabetic neuropathy. In socioeconomically disadvantaged groups, there is limited access to healthy food, health care, and, in some cases, safe places for physical activity. These are factors that may not be well seen in the health care setting, yet they tip the scales towards more diabetes cases and less favorable diabetic outcomes and more insulin resistance [20]. In the next 100 years, strategies to improve care access and nutritional access will pave the way to disease prevention, even while discoveries in genetics and biologics open up new frontiers.

\section{Conflicts of Interest}

The authors declare no conflicts of interest regarding the publication of this paper.

\section{References}

[1] Skljarevski, V. and Lledo, A. (2006) Diabetic Neuropathies. Archives of Neurology, 
63, 1502-1504. https://doi.org/10.1001/archneur.63.10.1502

[2] Hurst, G.C. (1979) Michinaga's Maladies. A Medical Report on Fujiwara No Michinaga. Monumenta Nipponica, 34, 101-112. https://doi.org/10.2307/2384283

[3] Skljarevski, V. (2007) Historical Aspects of Diabetic Neuropathies. Humana Press, Totowa, 1-5. https://doi.org/10.1007/978-1-59745-311-0_1

[4] Garland, H. (1960) The Neurological Complications of Diabetes Neurological Complications of Diabetes Mellitus: Clinical Aspects. Proceedings of the Royal Society of Medicine, 53, 137-141. https://doi.org/10.1177/003591576005300210

[5] Tesfaye, S., Boulton, A.J.M., Dyck, P.J., Freeman, R., Horowitz, M., Kempler, P., Lauria, G., Malik, R.A., Spallone, V., Vinik, A., et al. (2010) Diabetic Neuropathies: Update on Definitions, Diagnostic Criteria, Estimation of Severity, and Treatments. Diabetes Care, 33, 2285-2293. https://doi.org/10.2337/dc10-1303

[6] Schott, B. (1962) Neurological Complications of Diabetes. https://pubmed.ncbi.nlm.nih.gov/13909194/

[7] London, Z.N. (2020) A Structured Approach to the Diagnosis of Peripheral Nervous System Disorders. Peripheral Nerve and Motor Neuron Disorders, 26, 1130-1160. https://doi.org/10.1212/CON.0000000000000922

[8] Rundles, R.W. (1945) Diabetic Neuropathy General Review with Report of 125 Cases. Medicine, 24, 111-160. https://doi.org/10.1097/00005792-194505000-00001

[9] Callaghan, B.C., Little, A.A., Feldman, E.L. and Hughes, R.A. (2012) Enhanced Glucose Control for Preventing and Treating Diabetic Neuropathy. Cochrane Database of Systematic Reviews, Article No. CD007543 https://doi.org/10.1002/14651858.CD007543.pub2

[10] Gemignani, F. (2009) Acute Painful Diabetic Neuropathy Induced by Strict Glycemic Control ("Insulin Neuritis"): The Old Enigma Is Still Unsolved. Biomedicine \& Pharmacotherapy, 63, 249-250. https://doi.org/10.1016/j.biopha.2009.01.002

[11] Oyibo, S.O., Prasad, Y.D.M., Jackson, N.J., Jude, E.B. and Boulton, A.J.M. (2002) The Relationship between Blood Glucose Excursions and Painful Diabetic Peripheral Neuropathy: A Pilot Study. Diabetic Medicine, 19, 870-873. https://doi.org/10.1046/j.1464-5491.2002.00801.x

[12] Dejgaard, A. (1998) Pathophysiology and Treatment of Diabetic Neuropathy. Diabetic Medicine, 15, 97-112. https://doi.org/10.1002/(SICI)1096-9136(199802)15:2<97::AID-DIA523>3.0.CO;2-5

[13] Rahman, M.H., Jha, M.K. and Suk, K. (2016) Evolving Insights into the Pathophysiology of Diabetic Neuropathy: Implications of Malfunctioning Glia and Discovery of Novel Therapeutic Targets. Current Pharmaceutical Design, 22, 738-757. https://doi.org/10.2174/1381612822666151204001234

[14] Tavakoli, M., Mojaddidi, M., Fadavi, H. and Malik, R.A. (2008) Pathophysiology and Treatment of Painful Diabetic Neuropathy. Current Pain and Headache Reports, 12, 192-197. https://doi.org/10.1007/s11916-008-0034-1

[15] Veves, A., Backonja, M. and Malik, R.A. (2008) Painful Diabetic Neuropathy: Epidemiology, Natural History, Early Diagnosis, and Treatment Options. Pain Medicine, 9, 660-674. https://doi.org/10.1111/j.1526-4637.2007.00347.x

[16] Pop-Busui, R., Boulton, A.J., Feldman, E.L., Bril, V., Freeman, R., Malik, R.A., Sosenko, J.M. and Ziegler, D. (2016) Diabetic Neuropathy: A Position Statement by the American Diabetes Association. Diabetes Care, 40, 136-154. https://doi.org/10.2337/dc16-2042

[17] Çakici, N., Fakkel, T.M., Neck, J.W.V., Verhagen, A.P. and Coert, J.H. (2016) Sys- 
tematic Review of Treatments for Diabetic Peripheral Neuropathy. Diabetic Medicine, 33, 1466-1476. https://doi.org/10.1111/dme.13083

[18] Liampas, A., Rekatsina, M., Vadalouca, A., Paladini, A., Varrassi, G. and Zis, P. (2020) Non-Pharmacological Management of Painful Peripheral Neuropathies: A Systematic Review. Advances in Therapy, 37, 4096-4106.

https://doi.org/10.1007/s12325-020-01462-3

[19] Tuso, P. (2014) Prediabetes and Lifestyle Modification: Time to Prevent a Preventable Disease. The Permanente Journal, 18, 88-93.

https://doi.org/10.7812/TPP/14-002

[20] Gary-Webb, T.L., Giachello, A.L.M., Maier, K. and Skrabak, H. (2014) Socioecological Determinants of Prediabetes and Type 2 Diabetes: Agenda for Action. Clinical Diabetes, 32, 140-143. https://doi.org/10.2337/diaclin.32.3.140 